\title{
Design and implementation of automatic obstacle avoidance car control system
}

\author{
Hu Yanan \\ Mechanical and electrical technology department, Xijing University, Shaanxi Xi'an, China \\ 2664961273@qq.com
}

Keywords: obstacle avoidance car, ultrasonic sensor, motor driver, PWM

\begin{abstract}
In order to control the accuracy of the car during operation, to ensure that the car is safe and stable in the running process, using the STC89C52 as the control core, the car hardware selection, drawing process flow chart. Finally, through the combination of software and in-kind, using modular design ideas. The control system for debugging several times to achieve the car from the obstacle to the cycle of detection, display, alarm, automatic obstacle avoidance, variable speed driving, and automatic parking, reversing. So that the speed of the car can be controlled, in the process of moving speed will not be too fast, and obstacles can be maintained at a safe distance.
\end{abstract}

\section{Introduction}

With the development of automation technology, automation begins to develop from simple system control to complex system control and advanced intelligent control, and to various fields. Intelligent car is based on the automobile as the background, including automatic control, sensor technology, computer, machinery and other disciplines design. Intelligent car integrates complex integrated system, which can realize environment perception, self-planning and self-decision functions[1]. It can make full use of computer, sensor, information, communication, artificial intelligence and automatic control technology advantages, is a typical high-tech complex.

\section{Determination of system plan}

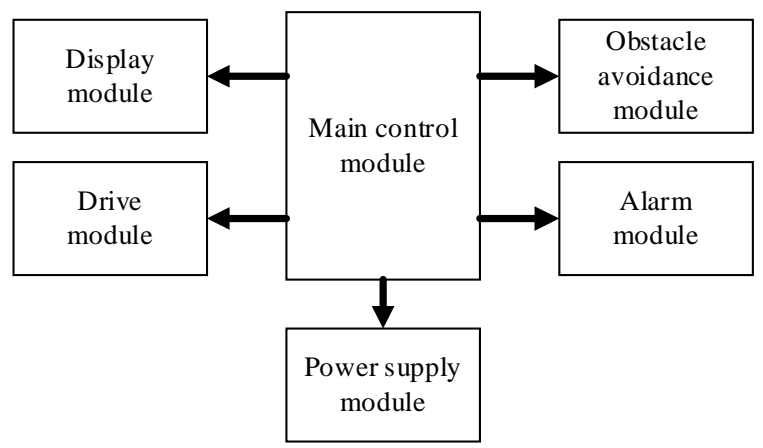

Fig. 1. General block diagram of system composition 
Using modular design, intelligent car is composed of the following parts: power module, main control module, obstacle avoidance module, drive module, display module, alarm module[2]. The overall system block diagram is shown in Figure 1.

\section{Determination of system plan}

As shown in Figure 2, obstacle avoidance car with steering gear can realize steering, but also can apply appropriate control methods to achieve obstacles in front of the car detection. The steering gear does not need the drive circuit, only needs one motor drive module to drive two DC motors, can save the hardware[3]. The hardware selection of obstacle avoidance trolley is shown in Table 1.

The L298N chip controls the speed of the trolley by controlling the pulse duty ratio algorithm. This kind of speed regulation mode has the advantages of excellent speed regulation characteristics, smooth adjustment, wide speed regulation range, large carrying capacity, can withstand frequent load impact, and can also realize frequent stepless fast start, braking and reversal.

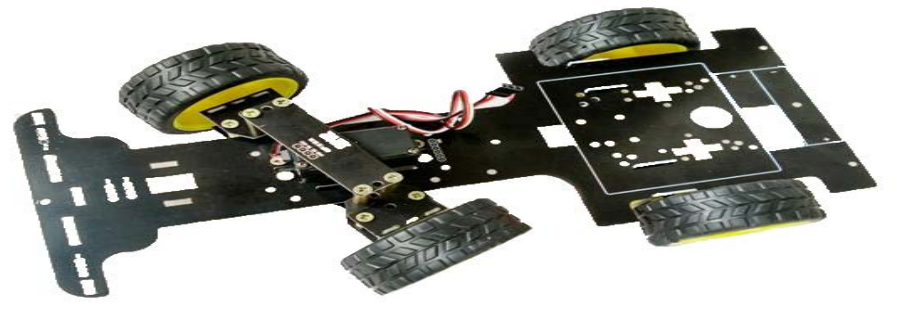

Fig. 2. Physical map with steering gear car

Table 1. Main components selection of each module

\begin{tabular}{|c|c|c|}
\hline Module & Main components & Function \\
\hline Main control module & STC89C52 & Control core \\
\hline $\begin{array}{c}\text { Obstacle avoidance } \\
\text { module }\end{array}$ & $\begin{array}{c}\text { HC-SR04 ultrasonic sensor; } \\
\text { Futaba s3003 Steering engine }\end{array}$ & Detect obstacles around the car \\
\hline $\begin{array}{c}\text { Display module } \\
\text { Trolley frame } \\
\text { module }\end{array}$ & $\begin{array}{c}\text { Two wheel drive type with } \\
\text { steering gear }\end{array}$ & $\begin{array}{c}\text { Play a supporting role, car structure } \\
\text { distance is displayed }\end{array}$ \\
\hline Alarm module & Buzzer & Early warning function \\
\hline $\begin{array}{c}\text { Power supply } \\
\text { module }\end{array}$ & 18650 series power supply & Provide motor operation power \\
\hline Drive module & L298N chip & $\begin{array}{c}\text { High voltage, high current and high } \\
\text { response frequency }\end{array}$ \\
\hline
\end{tabular}

\section{Hardware circuit design}

\subsection{Circuit design of obstacle avoidance module}

The Trig pin of HC-SR04 is connected with the P3.3 pin of MCU, and the high level above 10us is triggered by the P3.3 pin of MCU to drive HC-SR04. Then, the module will automatically send out eight $40 \mathrm{KHz}$ pulse signals, the module automatically detects the echo signal, and the microcontroller external interrupt trigger pin connected to the HC-SR04 Echo foot, to detect whether there is echo generated [4]. 


\subsection{Display module circuit design}

In a microcomputer application system, a 4-bit integrated LED display is used, and a P0 port is used as the segment output port of the display. Since the P0 port is an 8-bit bidirectional I/O port with an open drain, there is no pull-up resistance in it, a $10 \mathrm{~K}$ pull-up resistance must be added when it is to be used as the output and drive the load[5]. The P2 port is used as the selected terminal of the display, supplemented by the 8550 PNP transistor.

\subsection{Alarm module circuit design}

The buzzer operates with the P3.5 port of the single chip microcomputer and the 8550 PNP triode as the drive and the current limiting resistance. P3.6 and P3.7 control two LED lights respectively, and the hardware circuit is the same as that of the buzzer.

\subsection{Power module circuit design}

A 7805 regulator is used with a 9V dry cell input. A 100uF capacitor is connected in parallel at both ends of the 7805 regulator, and a $0.1 \mathrm{uF}$ capacitor is connected in parallel at the output end of the 7805 regulator, which can filter, reduce noise and stabilize voltage[6].

\subsection{Driving module circuit design}

The ENA and ENB are connected to the P1.0 and P1.1 ports of the single chip microcomputer respectively, and the PWM signal is controlled by the single chip microcomputer; the OUT1 and OUT2 are connected to P1.2 and P1.3 respectively to control the left motor; the OUT 3 and OUT4 are connected to P1.4 and P1.5 respectively to control the right motor; the ISENA and ISENB are the feedback terminals, grounded when not in use[7].

\section{Software design}

Software design, the use of modular design ideas, modular design advantages:

(1) each module is written separately, debugged and so on, which is relatively simple for learners to learn.

(2) modules can be called arbitrarily by different tasks at different times.

In the modular programming, according to the idea of integrity, the main function of the program is to initialize the various parts, and then integrate the various modules. The flow chart of the main program is shown in Figure 3.

The main procedure is as follows:

void main()

\{

$\mathrm{EA}=1$;

$\mathrm{TMOD}=0 \mathrm{x} 11$

T2MOD $=0 ; \quad$ // Set timer 2

$\mathrm{T} 2 \mathrm{CON}=0$;

PT2=1; $\quad$ // Priority of timer 2

$\mathrm{IPH}=0 \times 20$;

TH0 $=0$; // External interrupt timer initialization

TLO $=0$;

RCAP2H=(65536-100)/256; // Generating steering pulse width 
RCAP2L $=(65536-100) \% 256$;

TH2=RCAP2H;

TL2=RCAP2L;

TH1=(65536-100)/256; // Digital tube scanning, motor PWM, timing of ultrasonic cycle initialization

TL1=(65536-100)\%256;

ET0=1; // External interrupt timer

ET1=1; // The cycle of ultrasonic work

ET2=1; // PWM of motor

$\mathrm{EX} 0=1 ; / /$ External interrupt 0

IT0 $=0$; // External interrupt 0 low level trigger

TR2=1; // Turn on timer 2

serve_dj=14; // Steering gear in the middle

while(delay $1<=4000$ ); // Waiting for steering gear to return to center - 400ms

TR2 $=0$;

TR1=1;

duty_cycle=3; // Initialization duty cycle

start_ultrasonic();

while(1)

\{

\}

\}

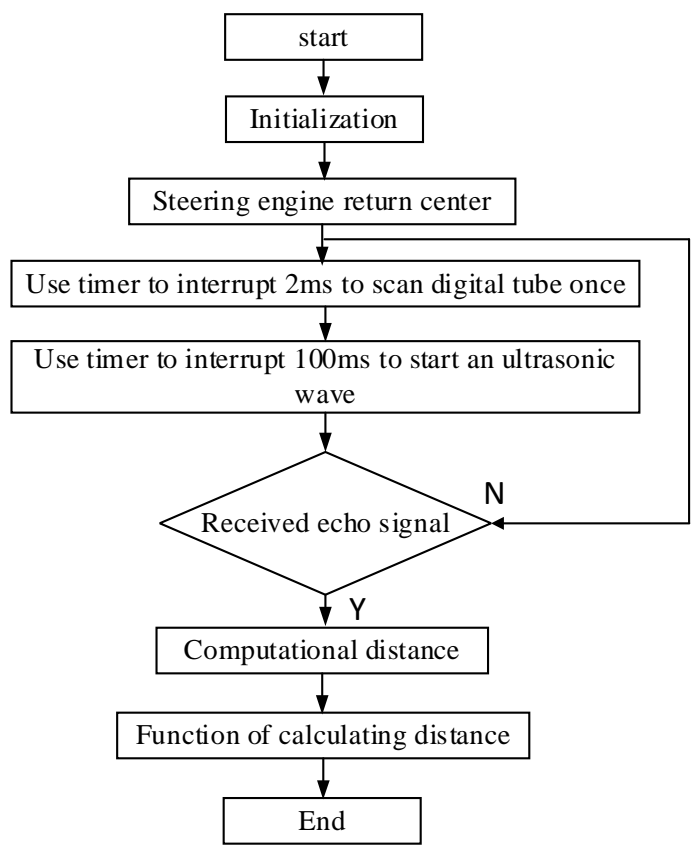

Fig. 3. Main program flow chart

From the front motor drive hardware circuit, we can see that the motor drive chip is L298N, according to the L298N chip manual can be obtained table 2, ENA, IN1, IN3 and ENB, IN2, IN4 take different levels of state combination, you can adjust the two motor forward and backward[8]. Take ENB, IN2, IN4 for example, when ENB $=0$, the motor stops rotating; when ENB $=1$, the state of the motor depends on different combinations of IN2, IN4, IN2IN4 $=00$ or IN2IN4 $=11$, the motor brakes; IN2IN4 = 01 when the motor is positive; IN2IN4 = 10 when the motor reverses. 
Table 2. L298N motor working status chart

\begin{tabular}{|c|c|c|c|}
\hline ENA/ENB & IN1/IN3 & IN2/IN4 & Motor working state \\
\hline 0 & $\mathrm{X}$ & $\mathrm{X}$ & Stop \\
\hline 1 & 0 & 0 & Braking \\
\hline 1 & 0 & 1 & Positive rotation \\
\hline 1 & 1 & 0 & Reversal \\
\hline 1 & 1 & 1 & Braking \\
\hline
\end{tabular}

HC-SR04 ultrasonic sensor outputs high-level pulse signal, and can not provide time and distance information itself. The idea of this design is that when the ultrasonic module receives the signal, it will generate a corresponding high-level pulse according to the distance from the module to the obstacle, then use the inverter to reverse it, trigger the external interrupt 0 , turn on the timer in the external interrupt 0 processing function, start timing, when the ultrasonic return signal becomes low-power. Flat pulse, stop the time, and then use software to calculate the distance. Because the precision of the ultrasonic module is $0.3 \mathrm{~cm}$, it is difficult to read the decimal point of the floating-point number. Therefore, this design will magnify the calculated number by 10 times, and then take the module, making the difficulty smaller.

\section{Test result}

Fig. 4 shows the waveform of the ECHO output when the obstacle is 10CM away from the ultrasonic module, and Fig. 5 shows the waveform of the ECHO output when the obstacle is not in front of the ultrasonic module.

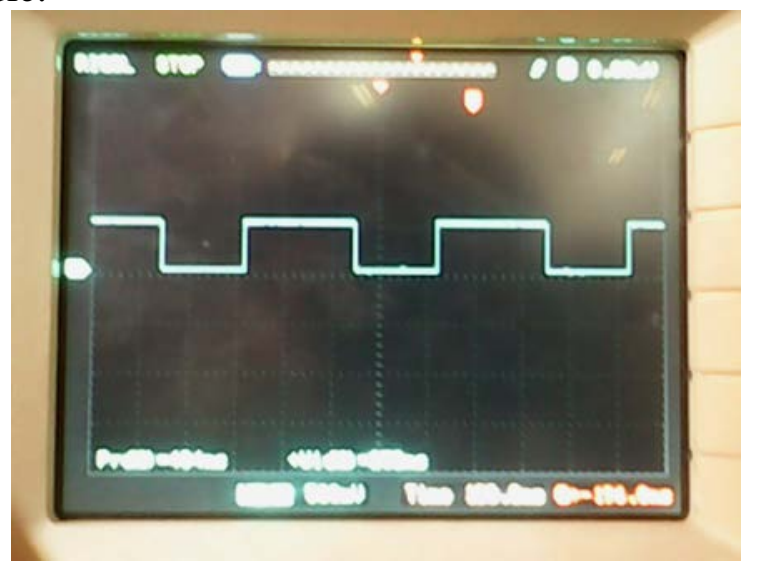

Fig. 4. ECHO terminal waveform at $10 \mathrm{~cm}$ distance

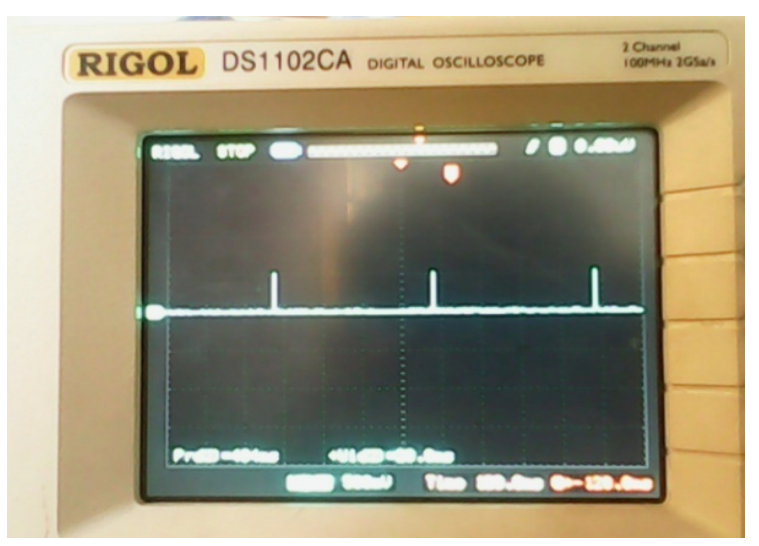

Fig. 5 waveforms of ECHO terminals without obstacles 


\section{Conclusions}

The obstacle avoidance system of intelligent car based on STC89C52 has the advantages of simple design, stable performance, low cost, and measurement accuracy to meet the requirements of the obstacle avoidance system. The measuring precision of the ultrasonic distance module can reach $0.3 \mathrm{~cm}$, the minimum measuring distance is $2 \mathrm{~cm}$, and the maximum measuring distance is $400 \mathrm{~cm}$. The ultrasonic obstacle avoidance system can set the specific distance of obstacle avoidance, and the range of obstacle avoidance is wide, so it is widely used. In the actual test, the car runs steadily, can avoid obstacles very well, and the car is not easily disturbed by the outside world to avoid obstacles, and achieved good results.

\section{References}

[1] LU Chun-yu, WANG Yan. Design of intelligent obstacle avoidance trolley system based on single chip microcomputer [J]. Electronic world, 2014, 27(17):146-147.

[2] JI Hong-xu. Design of intelligent obstacle avoidance car based on STC89C52 single chip microcomputer [J]. Vocational Technology, 2011, 17(8): 141-142.

[3] LI Ming-ping. Design and research of robot obstacle avoidance module [D]. Beijing: China University of Petroleum, 2007.

[4] HU Sheng-bin, LUO Jun, GONG Zhen-bang. Ultrasonic ranging system for mobile robot obstacle avoidance [J]. Mechatronics, 2009, 36(7): 198-200.

[5] WANG Jing. Research on motion control technology of intelligent car [D]. Wuhan: Wuhan University of Technology, 2009.

[6] LU Wei. Design and implementation of intelligent car obstacle avoidance system [D]. Nanchang: Nanchang University, 2012.

[7] YUAN Xin-na, CHENG Bing. Ultrasonic ranging system based on single chip microcomputer [J]. Ordnance automation, 2005,24 (5): 77-79.

[8] SHU Xiu-lan. Design of ultrasonic ranging system based on AT89C2051 single chip microcomputer [J]. digital technology and application, 2013, (5), 159-161. 